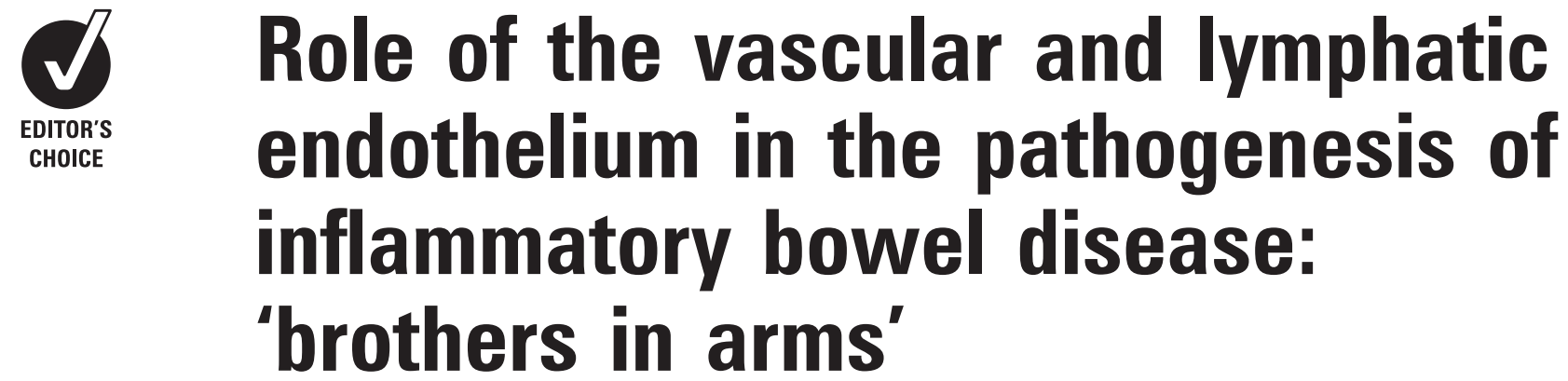

\title{
Silvio Danese
}

Correspondence to

Dr Silvio Danese, Head, IBD

Unit, Division of

Gastroenterology, IRCCS Istituto

Clinico Humanitas, Via Manzoni

56, 20089, Rozzano, Milan,

Italy; sdanese@hotmail.com

Published Online First

6 January 2011

\begin{abstract}
The 'IN' of chronic inflammation-that is, the mechanisms of cell entry into the intestinal mucosa, bacterial and foreign antigen invasion, angiogenesis, and the control of gut inflammation through intestinal microvasculature-has received a great deal of attention in studies of the pathogenesis of inflammatory bowel disease (IBD). This has resulted in the validation of several targets for the treatment of experimental inflammation - both on immune and non-immune cells-some of which have translated into effective treatments for patients with IBD. An important aspect of this has been our growing understanding of the role the intestinal vascular microcirculation plays in the initiation and perpetuation of the inflammatory process, by regulating the migration of leucocytes into the interstitial space. However, it is becoming increasingly clear that it is also important to focus on the 'OUT' of chronic inflammation - that is, the lymphatics and their role in controlling tissue oedema, leucocyte exit, bacterial antigen and inflammatory chemokine clearance. As our understanding of the lymphatics and the role they play grows, another rich source of non-immune cell targets for therapeutic intervention is gradually being revealed. This article describes current knowledge of the roles played by the vascular and lymphatic endothelium throughout the gut in the pathogenesis of IBD, and how this differs from their role under physiological conditions, as well as discussing current and future therapeutic targets that have been identified.
\end{abstract}

\section{INTRODUCTION}

The study of inflammatory bowel diseases (IBD) has largely focused on the roles played by the classical components of the immune system, a unidirectional viewpoint that has dominated the study of Crohn disease (CD) and ulcerative colitis (UC), the two major forms of IBD. However, as the emphasis of studies has broadened to encompass environmental factors, intestinal microbial flora, the tissue response and the underlying genetics, an increasing number of reports have called attention to the significant contribution of non-immune cells such as epithelial, endothelial, mesenchymal, nerve and vascular cells, platelets and the extracellular matrix to the pathogenesis of IBD. ${ }^{1-3}$ These cell types are not only able to perform many of the functions traditionally attributed to classical immune cells, ${ }^{4}$ but they also secrete or express on their surface molecules involved in the immune response, particularly those of innate immunity. ${ }^{2-8}$ Indeed, it is becoming clear that there is a highly integrated network of immune and non-immune cells, involving many different types of interaction. ${ }^{1-9}$ This adds a further layer of complexity to our current understanding of the mechanisms underlying the pathogenesis of IBD.

An extensive body of research has demonstrated that the intestinal vascular microcirculation plays a central role in both the initiation and perpetuation of the inflammatory process. Its ability to regulate both the type and number of leucocytes that migrate into the interstitial space places it in a unique and key position to govern the infiltration of leucocytes into the gut. ${ }^{10-12}$ During inflammation, the phenotype of activated vascular endothelial cells (VECs) includes leakiness, leucocyte adhesiveness and procoagulant activity, and, further down the line, angiogenesis. ${ }^{13} 14$ However, while research has devoted a lot of attention to the investigation of the mechanisms of inflammation and how leucocytes arrive in the inflamed tissue, the mechanisms that mediate the resolution of inflammation, including the exit of leucocytes from the inflamed tissue into the lymphatics, have only recently begun to be elucidated. ${ }^{15}$

Although the role played by the lymphatic endothelium has not received the same level of attention as that of the vascular endothelium, the very recent development of techniques to isolate and culture lymphatic endothelial cells (LECs) in vitro has enabled significant progress in the study of the lymphatics by allowing the characterisation of LECs. Unique markers that differ from VECs have been identified, as well as mechanisms through which they are able to mediate postinflammatory clearance of both leucocytes and chemokines. However, few data have been published thus far specifically describing LECs in the gut, either under physiological conditions or in the presence of inflammation. ${ }^{16} 17$

In this review, I describe the current knowledge of the roles played by the vascular and lymphatic endothelium throughout the gut in the pathogenesis of IBD, and how this differs from their role 
under physiological conditions. I will also discuss the potential of the two endothelial systems as distinct and disparate key targets for therapeutic intervention.

\section{MICROVASCULAR ENDOTHELIUM}

The highly specialised cellular system that forms the intestinal microvasculature performs a wide range of biological tasks that are the crucial underpinning of multiple physiological processes, such as blood flow, the flow of nutrients, tissue homoeostasis, and cell trafficking and distribution. In addition, the vascular endothelium has also been implicated in pathological processes such as inflammation. Studies of the functions performed by VECs have been significantly advanced by the availability of cultured human umbilical vein endothelial cells (HUVECs). However, it has also become clear that VECs have many tissue-specific characteristics, and stress and inflammatory stimuli can change the expression patterns of adhesion molecules, activate unique sets of genes, and trigger distinct chemokine secretory patterns. ${ }^{18} 19$ This tissue specificity of VECs limits the information that can be gained from the study of HUVECs.

For the study of VECs in the mucosa of the gut, this deficiency drove the development of protocols for routine isolation and long-term culture of pure populations of human intestinal microvascular endothelial cells (HIMECs). ${ }^{19}$ The importance of this development to the study of intestinal inflammation was exemplified by the demonstration of unique patterns of leucocyte adhesion and growth for HIMECs compared with HUVECs. This demonstration also supports the belief that tissuespecific mediators and transcription factors contribute to the induction or maintenance of a specific tissue VEC profile. ${ }^{11} 19-21$

\section{Leucocyte recruitment}

Recruitment of leucocytes from the vascular circulation into inflamed tissues is an important step in the inflammatory response which is entirely regulated by the microvascular endothelium. In response to activation by cytokines and other inflammatory mediators, the microvascular endothelium expresses cell adhesion molecules (CAMs) and chemokines that enhance interactions with leucocytes and their subsequent recruitment. ${ }^{12} 2223$ The leucocyte extravasation cascade involves multiple steps, including tethering/rolling, activation, adhesion, spreading and transmigration, and has been extensively reviewed elsewhere. ${ }^{24-26}$ Many in vitro and in vivo studies have focused on the contribution of molecular elements at each step of the extravasation cascade to the pathogenesis of IBD. $^{27}$

Leucocyte homing to the gut is mainly mediated by the CCR9 chemokine receptor, which binds to CCL25 on the surface of the ileal endothelium. ${ }^{28-30}$ Subsequent adhesion is then supported by the binding of integrins expressed on the surface of the leucocytes to the CAMs expressed on endothelial cells (figure 1). Indeed, adhesion is the crucial event that determines which leucocytes will ultimately migrate into the tissues. In the gut, this is mainly mediated by CD11a/CD18 on the leucocytes binding to intercellular adhesion molecule (ICAM)1 , or by binding of $\alpha 4 \beta 1$ or $\alpha 4 \beta 7$ to vascular cell adhesion molecule (VCAM)-1 and mucosal addressin cell adhesion molecule (MAdCAM)-1. ${ }^{23-27}$ The importance of this pathway is reflected in the fact that it has been a rich source of targets for therapeutic intervention. Indeed, one drug targeting this pathway has been approved by the Food and Drug Administration for the treatment of moderateto-severe CD: natalizumab is a recombinant humanised IgG4 directed against a4 integrins. New drugs that target leucocyte-endothelial interactions are now under development, including vedolizumab (a humanised antibody to $\alpha_{4} \beta_{7}$ integrin), PF-547659 (a monoclonal antibody to MAdCAM-1), rhuMab $\beta_{7}$ (an anti $\beta_{7}$ integrin) and CCX282-B (an oral CCR9 inhibitor) (figure 1).

In patients with IBD, the homing of leucocytes to the microvasculature is profoundly altered. Indeed, HIMECs isolated from chronically inflamed areas of the intestine of patients with IBD have a significantly enhanced capacity to adhere leucocytes compared with HIMECs either from uninflamed areas from the same patients or from controls. ${ }^{19} 31$ In accordance with this, in the intestinal microvasculature of patients with IBD, there is enhanced expression of the gut-specific homing model MAdCAM-1, which plays a major role in the recruitment of the gut-specific $\alpha 4 \beta 7$ integrin-expressing leucocytes into the mucosal immune compartment. ${ }^{32} 33$ In addition, increased expression of ICAM-1 and VCAM-1 further ensures continuous recruitment of leucocytes to the gut. ${ }^{34}$

Characterisation of HIMECs has demonstrated that other molecules are also specifically upregulated in the endothelium of patients with IBD. Fractalkine is a unique $\mathrm{CX}_{3} \mathrm{C}$ chemokine that acts as an adhesion molecule and is upregulated in the endothelium of patients with IBD. ${ }^{35}$ Moreover, patients with IBD have greater numbers of circulatory $\mathrm{T}$ cells that express the $\mathrm{CX}_{3} \mathrm{C}$ chemokine receptor CX3CR1 than controls. ${ }^{35}$ Fractalkine could therefore be an important mediator of interactions between VECs and leucocytes under conditions of intestinal inflammation.

Finally, intestinal endothelial cells also express CD40, an important immune costimulatory molecule for T cells and platelets, which express CD40 ligand (CD40L). ${ }^{34}$ In the endothelium of the mucosa of patients with IBD, CD40 is activated by binding to CD40L, inducing the cells that express CD40L to produce inflammatory chemokines such as RANTES (regulated upon activation, normal T cell expressed, and secreted) and cell adhesion molecules, fostering and amplifying intestinal inflammation. ${ }^{37-40}$ Furthermore, leucocyte and platelet recruitment to the inflamed intestine is decreased in mice deficient in CD40 and CD40L. This indicates that the CD40-CD40L signalling pathway could be a relevant target for therapeutic 


\section{LEUCOCYTE ADHESION}

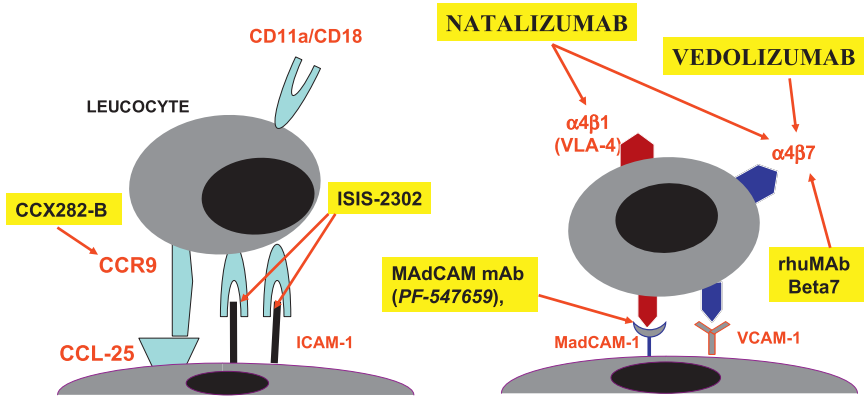

ACTIVATED INTESTINAL MICROVASCULAR ENDOTHELIAL CELLS

Figure 1 Cell adhesion molecules involved in the multistep paradigm of leucocyte recruitment, and therapeutic targets that have been developed or are under development for the treatment of patients with inflammatory bowel disease. ICAM-1, inter cellular adhesion molecule-1; VCAM-1, vascular cellular adhesion molecule-1; MadCAM-1, mucosal addressin cellular adhesion molecule-1; VLA, very late antigens.

intervention to inhibit the recruitment of leucocytes to the inflamed intestine. ${ }^{36-384041}$

In addition to studying specific molecular pathways, mechanisms that lead to aberrant activation of endothelial cells are also now being characterised. In addition to the proinflammatory cytokines that enhance the expression of ICAM-1, VCAM-1 and MAdCAM-1, several other mediators have recently been described. For instance, nitric oxide (NO) is an inflammatory mediator for which a role in IBD has clearly been established. ${ }^{42}$ Generation of NO was therefore investigated in HIMECs, to determine whether this alternative pathway also influences the activation of VECs in the gut, including their capacity to bind circulating leucocytes. Generation of $\mathrm{NO}$ occurred in control HIMECs through both constitutive endothelial NO synthase (eNOS or NOS3) and inducible $\mathrm{NO}$ synthase (iNOS or NOS2); however, expression of the mRNA for iNOS was lost in HIMECs derived from patients with IBD, which corresponded to a decrease in NO generation and enhanced leucocyte binding. ${ }^{43} 44$ Finally, an increase in arginase activity is also observed in HIMECs exposed to an inflammatory milieu, which may contribute to the decrease in production of $\mathrm{NO}^{45}$

HIMECs are also activated by homocysteine, a known player in microvascular inflammation in patients in IBD. Activation of VECs by homocysteine alone or in combination with tumour necrosis factor (TNF) $\alpha$ (which synergises with homocysteine), upregulates expression of VCAM-1, production of monocyte chemotactic protein-1 (MCP-1), and phosphorylation of p38 mitogenactivated protein kinase (MAPK). ${ }^{46}$

The MAPK signal transduction pathways are important players in a variety of cellular processes, including regulation of expression from genes for CAM and cytokine production. Scaldaferri et al have recently studied the functional role of the MAPK p38, p42/44 and JNK in the regulation of lymphocyte extravasation. The phosphorylation levels of MAPK are higher in both the fibroblasts and endothelium of the mucosa of patients with IBD. ${ }^{47}$ They also play an important role in regulation of expression of CAMs, chemokine production by HIMECs, and extravasation of lymphocytes into the tissue. ${ }^{47}$

Given the important role they play in processes central to the development and maintenance of chronic inflammation, MAPK inhibitors could be potentially important targets for anti-inflammatory therapeutics. ${ }^{48}$ Indeed, potent anti-inflammatory activity has been demonstrated for several MAPK inhibitors. For example, pyridinyl imidazole compounds, which can reduce synthesis of inflammatory cytokines, ${ }^{48}$ are selective inhibitors of p38 MAPK. ${ }^{48}$ In addition, a potent inhibitor of JNK/p38 MAPK, CNI-1493 (a synthetic guanylhydrazone), has shown clinical benefit and mucosal healing in clinical trials in patients with severe CD. ${ }^{49}$ However, several side effects related to liver and central nervous system toxicity have been observed in clinical trials with MAPK inhibitors, as a result of which the development of these agents has been suspended. Given that p38 MAPK plays such a pivotal role in processes other than inflammation, it is not surprising to find that their systemic use results in undesirable side effects. On the other hand, local inhibition of MAPK activation in mucosal endothelial cells of patients with IBD could specifically inhibit the aberrant homing of leucocytes to the gut. This promising possibility warrants the investigation of drug carriers that can specifically target MAPK inhibitors to inflamed tissues in such patients.

Studies of these pathways have already revealed many potential, as well as some actual, targets for therapeutic intervention, and it is likely that many more will be revealed.

\section{Angiogenesis}

Chronic inflammation is always accompanied by angiogenesis, the growth of new blood vessels, and indeed it is becoming clear that angiogenesis and inflammation are intertwined processes. ${ }^{50}$ Extensive angiogenesis and remodelling of the microvasculature are intrinsic to the tissue remodelling that occurs in inflamed intestine in patients with IBD. ${ }^{51} 52$ Although angiogenesis and remodelling are distinct phenomena that occur in response to different triggers, they often occur together in the tissues of patients with IBD, and both involve proliferation of endothelial cells.

The processes of inflammation and angiogenesis are linked on a number of levels. Hypoxia in inflammatory tissues is an important proangiogenic stimulus, as it upregulates angiogenic factors such as vascular endothelial growth factor (VEGF), fibroblast growth factor (FGF), TNF $\alpha$ and hypoxiainducible factor $1 .{ }^{51} 52$ In addition, extravasated plasma fibrinogen is involved in stimulation of neovascularisation. ${ }^{51} 52$ Inflammatory cells, such as macrophages, lymphocytes, mast cells and fibroblasts, produce diverse angiogenic factors that stimulate vessel growth. ${ }^{51}$ Activated platelets have also recently been identified as potent inducers 
of intestinal angiogenesis, ${ }^{53}$ and shear stress on the endothelium due to increased blood flow may stimulate angiogenesis. $^{54}$ Finally, recently, an active contribution of the bacterial flora to the induction of VEGF-A and angiogenesis has been demonstrated. $^{5556}$

Initially, the prevalent changes to the vascular endothelium are functional, including dilation, increased permeability, activation of the endothelium and diapedesis. The next step is structural changes, with capillary and venule remodelling and proliferation of endothelial cells. ${ }^{57}$ Indeed, in chronic inflammatory disorders, tissue damage and repair continue concurrently. ${ }^{58}$ With time, the endothelial cells in the inflamed capillaries respond to locally produced angiogenic factors and start to multiply, forming permanent remodelled vessels. ${ }^{58}$ This anatomical expansion of the microvascular bed combines with its increased activation state to foster further influx of inflammatory cells, a cyclical step that results in chronic co-dependence of angiogenesis and inflammation.

VECs that participate in the angiogenic process display an activated cell surface molecular pattern not found on resting vessels, with altered expression of endothelial adhesion molecules, ${ }^{57} 58$ and increased concentrations of soluble adhesion molecules ${ }^{24}$ observed in intestinal biopsy specimens from patients with IBD. Angiogenic vessels can be identified through the expression of specific integrins, in particular $\alpha_{v} \beta 3$ and $\alpha v \beta 5$; several receptors for angiogenic factors are also measurably upregulated. 65

Neoangiogenesis has been investigated in patients with $\mathrm{CD}$ and UC by quantifying the mucosal vascularisation state, assessing local expression of markers of angiogenesis, and determining the presence of functional proangiogenic activity in inflamed tissue from patients with IBD. ${ }^{59}$ The mucosal vascularisation state was quantified by counting the number of vessels that were positive for the vessel marker CD31. A significant increase in the number of vessels was found in tissues from affected areas of patients with IBD compared with controls, which suggests that active angiogenesis has taken place. ${ }^{59}$

Expression of the neoangiogenic marker $\alpha \mathrm{v} \beta 3$ by the mucosal microvasculature was most prominent in inflamed areas of the mucosa. Moreover, $\alpha_{v} \beta 3$ was upregulated in cultured HIMECs that were exposed to proinflammatory and proangiogenic factors that are overexpressed in inflamed tissue from patients with IBD - that is, TNF $\alpha$, VEGF, interleukin (IL)-8, and basic FGF (bFGF), which probably act in a complementary fashion. ${ }^{59}$ Along similar lines, the novel marker of endothelial junction remodelling, CD146, is highly expressed on VECs in intestinal biopsy specimens from patients with IBD, which is associated with decreased concentrations of the soluble form of CD146. ${ }^{60}$ In addition, increased serum and/or tissue concentrations of several proangiogenic factors, such as bFGF, VEGF-A, angiogenin, angiopoietins, substance $\mathrm{p}$ and corticotrophin-releasing hormone, have been reported in patients with active IBD and in experimental colitis. ${ }^{61-69}$ Indeed, mucosal extracts from patients with IBD exhibited an augmented capacity to induce dose-dependent migration of HIMECs, suggesting that the angiogenic-enriched mucosa in patients with IBD is functionally active.

The extent and complexity of the network of signals in which endothelial cells and their angiogenesis is involved is exemplified by the critical role of the CD40-CD40L pathway in immune-driven angiogenesis. ${ }^{34} 70$ In addition to the direct role that soluble CD40L plays in mucosal angiogenesis, ${ }^{70}$ inflammation-activated CD40L-expressing T cells may activate intestinal fibroblasts, causing them to release angiogenic cytokines that activate HIMEC angiogenesis. Indeed, mice that are deficient in CD40 or CD40L have impaired, pathological angiogenesis in the gut. ${ }^{70}$ This would point to a dual mechanism underlying CD40-dependent angiogenesis in the inflamed gut.

The complexity of the interactions between inflammation and angiogenesis in experimental colitis is underlined by the overlap between some common mediators. For instance, the angiogenic factor, VEGF-A, can induce expression of CAMs on the intestinal endothelium and promote adhesion of leucocytes in a similar manner to more classical inflammatory agents such as TNF $\alpha^{71}$ The importance of angiogenesis to the pathophysiology of experimental colitis has been demonstrated in both the dextran sodium sulfate (DSS)-induced and CD4 ${ }^{+}$CD45RB ${ }^{\text {high }}$ T cell transfer models of colitis. ${ }^{72}$ Upregulation of proangiogenic mediators such as matrix metalloproteinase 2 and 9, endothelial sphingolipid G-protein-coupled receptor 1, endoglin, prostaglandin-endoperoxide synthase 2, $\mathrm{TNF} \alpha$, chemokine (CXC) ligand 1, and hepatocyte growth factor, as well as downregulation of some antiangiogenic factors, including CD36 antigen and chromagranin A, was observed in both colitis models. Interestingly, the authors found differential regulation of numerous angiogenic, antiangiogenic and angiostatic genes between these two models, suggesting that angiogenesis occurs through different mechanisms in the two models: loss of angiogenic inhibition in the DSS model, and upregulation of proangiogenic mediators in the $\mathrm{CD}{ }^{+} \mathrm{CD} 45 \mathrm{RB}^{\text {high }}$ model. Both of these mechanisms could be potential sites for intervention for selective treatment of various forms of IBD. ${ }^{72}$

Indeed, angiogenesis has already proven to be an effective therapeutic target for the treatment of experimental colitis. In the IL-10 knockout model of colitis, blockade of $\alpha v \beta 3$ effectively ameliorated colitis and decreased the production of inflammatory cytokines. ${ }^{73}$ Similarly, inhibition of VEGF-A was effective in both the DSS-induced ${ }^{74}$ and trinitrobenzenesulfonate (TNBS)-induced ${ }^{75}$ models of colitis, improving histological inflammation and inhibiting mucosal cytokine production in both.

Several of the factors involved in pathological angiogenesis in chronically inflamed tissues are regulated at specialised lipid rafts known as 
caveolae. The major structural protein of these caveolae, caveolin-1 (Cav-1), is involved in the regulation of angiogenesis, ${ }^{76}$ and its expression is increased after the induction of DSS colitis. In Cav$1(-/-)$ mice or mice administered a Cav-1 inhibitory peptide, the colitis histopathology scores, vascular densities and concentrations of inflammatory infiltrates were significantly decreased after induction of colitis compared with wild-type mice. In addition, lower concentrations of leucocyte and platelet rolling and adhesion colitis were observed in these mice compared with wild-type mice. Interestingly, Cav-1(-/-) mice that received transplants of wild-type bone marrow had a lower colitis score than wild-type mice, while disease was not attenuated in wild-type mice that received Cav $1(-/-)$ bone marrow. In addition, mice that overexpress Cav-1 only in the endothelium developed disease similar to that of wild-type mice. These data indicate that endothelial Cav-1 may play a critical role in the regulation of colitis. In addition to its effects on disease severity, specific deletion or blockade of endothelial Cav-1 decreased vascular densities and angiogenesis scores. ${ }^{76}$

Natural antiangiogenic molecules such as thrombospondin-1 (TSP-1) have also consistently been found to control colitis-associated angiogenesis. Indeed, TSP-1 knockout mice have increased susceptibility to DSS-induced colitis, whereas blockade of TSP-1 decreases disease severity, thus reinforcing the therapeutic potential of targeting angiogenesis for colitis, ${ }^{77} 78$ as well as specifically implicating TSP-1 as a novel target for the treatment of IBD.

However, the translational therapeutic implications of these findings are limited by the consideration that angiogenesis is an essential aspect of the tissue healing process, and therefore any therapy that targets angiogenesis may have potentially serious side effects. Indeed, the colon cancer drug, bavacizumab, which targets human VEGF, induces ulcers and gastrointestinal bleeding as a deleterious side effect. A case report that described administration of bavacizumab to a patient with UC also reported this side effect. ${ }^{79}$ In addition, administration of the antiangiogenic sorafenib (a receptor tyrosine kinase inhibitor that inhibits VEGFR1, 2, 3) to a patient with UC also exacerbated the disease. ${ }^{80}$

Despite the promising data on the potential for blockade of angiogenesis, profound alteration of wound healing through inhibition of angiogenesis could be deleterious for IBD depending on the stage of disease. This has been highlighted by a study in which knockout of the VEGF homologue placental growth factor significantly worsened disease severity and morbidity in mice with DSS- or TNBSinduced experimental colitis. Furthermore, angiogenesis was significantly decreased compared with wild-type mice in which colitis had been induced. ${ }^{81}$

Nonetheless, potentially promising effects of promotion of wound healing through stimulation of angiogenesis were suggested by a study of the probiotic Bacillus polyfermenticus. When HIMECs were exposed to $B$ polyfermenticus conditioned
Box 1

Angiogenesis is a constant feature in both human and experimental IBD

- Many inflammatory mediators and cell types promote pathological angiogenesis

- Blockade of angiogenesis can be therapeutically beneficial

- Since angiogenesis is also relevant for tissue healing, its blockade could lead to mucosal ulcers depending on the disease stage

medium, cell migration, permeability and tube formations were increased, as well as production of IL-8. In addition, inoculation of mice with $B$ polyfermenticus facilitated their recovery from colitis, as well as increasing angiogenesis and production of IL-8 in the mucosal layer. $B$ polyfermenticus therefore promotes angiogenesis in the mucosa during recovery of mice from colitis, suggesting clinical utility of this probiotic for intestinal wound healing. ${ }^{56}$

These data indicate that the potential utility of inhibitors of angiogenesis may be specific to the stage of disease, but that the risks of targeting angiogenesis should be carefully evaluated when considering whether preclinical findings can translate into clinical utility.

\section{Coagulation}

That IBD is associated with both a hypercoagulable state and a prothrombotic condition has been demonstrated in the clinic and by laboratory researchers. Indeed, coagulation abnormalities are intrinsic to IBD, while a significant proportion of the morbidity and mortality in patients with IBD is caused by thromboembolic disease. ${ }^{5}$ This haemostatic risk appears to be unique to IBD among the chronic inflammatory diseases, as no increased risk has been observed in patients with rheumatoid arthritis and other chronic bowel diseases, such as coeliac disease.

Besides thromboembolism, coagulation plays an essential role in inflammation, as they are closely linked and interdependent processes. ${ }^{5} 82$ Under physiological conditions, the tissue microcirculation rests in an anticoagulant and anti-inflammatory state. However, when inflammation occurs, coagulation is activated and participates in the spreading of inflammation. Recently, novel and unexpected roles of haemostasis in the humoral and cellular components of immunity have been described. 582 For instance, platelets, which are typically considered as simply coagulative cells, play a role in the microcirculation of patients with IBD by fostering inflammation. ${ }^{83}$

The mucosa of patients with IBD also shows signs of both coagulation abnormalities and thromboembolic complications. This is exemplified by the fact that one of the earliest abnormalities that has been identified in the mucosa of patients with $\mathrm{CD}$ is the presence of platelet thrombi 
cross-linked with fibrin in the mucosal microvasculature. ${ }^{84}$ Other such crucial changes to the mucosal microvasculature have been identified in patients with IBD, including vascular injury, focal arteritis, fibrin deposition, micro-infarction and neoangiogenesis in patients with $\mathrm{CD},{ }^{85}$ as well as intracapillary clots in rectal biopsy specimens of patients with UC. ${ }^{86}$ Finally, injury and the resulting disruption of the endothelium could expose the subendothelial matrix, to which platelets are strongly attracted, further promoting the formation of micro thrombi.

Indeed, thrombi that adhere to the endothelium are easy to find in the microcirculation of patients with either UC or CD, using confocal microscopy. Both circulating and mucosal platelets express activation markers such as P-selectin and CD40L, and are able activate HIMECs in a CD40-dependent manner, resulting in increased surface expression of ICAM-1 and VCAM-1, enhanced production of IL-8, and increased adhesion of leucocyte endothelial cells. In addition, the activated platelets release many factors that probably play a role in colonic inflammation: RANTES binds to the endothelial cell surface to further promote leucocyte recruitment, while soluble CD40L, histamine, platelet activator factor (PAF) and cationic proteins may contribute to endothelial activation, barrier dysfunction and increased vascular permeability. ${ }^{36} 87$

A critical role for platelets has also been demonstrated in experimental animal models of colitis. DSS-induced colonic inflammation is marked by accumulation of platelets in colonic venules which coincides with enhanced adherence of leucocytes and worsening disease activity. ${ }^{87}$ Of these platelets, $\sim 20 \%$ are bound directly to endothelial cells, while $\sim 80 \%$ are attached to the surface of adherent leucocytes. ${ }^{88}$ After induction of colitis in mice with DSS, the expression of CD40 is increased on endothelial cells in the colonic microcirculation, while deficiency of either CD40 or CD40L attenuates leucocyte-endothelial cell adhesion. ${ }^{41}$ Platelet-associated CD40L (or its circulating soluble form) may also mediate interaction of platelets with both adherent leucocytes and the endothelium, as depletion of platelets significantly reduces the recruitment of leucocytes to the inflamed gut.

Another important receptor-ligand pair, P-selectin and P-selectin glycoprotein ligand-1 (PSGL-1), is also affected by induction of colitis with DSS. Expression of P-selectin is increased on the surface of platelets and endothelial cells, while PSGL-1 is increased on endothelial cells and leucocytes. The importance of the P-selectin interaction with PSGL-1 is reinforced by the demonstration that immunoblockade or genetic deletion of either significantly reduces the recruitment of both platelets and leucocytes after stimulation with $\mathrm{DSS}^{87} 88$ P-selectin on the surface of platelets also mediates binding to leucocytes, which constitutively express PSGL-1 in their surface; the overall increase in leucocyte adhesion therefore further serves to recruit platelets. ${ }^{89}$

Beside platelets, another major system that bridges inflammation and coagulation is the protein C (PC) system, ${ }^{90}$ which both participates in and controls mucosal microvascular inflammation in patients with IBD. ${ }^{91} 92$ The expression of the anticoagulant, thrombomodulin, and endothelial PC receptor is dramatically downregulated in microvessels of patients with IBD. ${ }^{92} 93$ Similarly, surface expression of thrombomodulin and endothelial PC receptor on HIMECs is downregulated by the inflammatory mediators, TNF $\alpha$ and IL-1 $\beta .^{92}$ In addition, the activation of $\mathrm{PC}$ that occurs constitutively under resting conditions in HIMECs is inhibited by stimulation with these proinflammatory cytokines.

On the other side of this equation, PC can also be potently anti-inflammatory, inhibiting the ability of TNF to upregulate CAMs and the secretion of chemokines from HIMECs. Activated PC was potently anti-inflammatory in cultured HIMECs, downregulating cytokine-dependent CAM expression and chemokine production, and inhibiting adhesion of leucocytes. In mice with DSS-induced colitis, activated PC ameliorated disease, with reduced weight loss, disease activity index, and histological colitis scores, as well as inhibition of leucocyte adhesion to inflamed intestinal vessels. ${ }^{92}$

In an experimentally induced model of extraintestinal thrombosis, the application of DSS enhanced thrombus formation. However, this could be attenuated by either the administration of activated PC, or overexpression of the endothelial PC receptor, while an antibody to activated PC enhanced thrombus formation. These data indicate a protective effect of activated PC against extraintestinal thrombosis, and further underline the complex link between inflammation and thrombosis. $^{94}$

These data suggest that the PC pathway is functionally impaired in the mucosal microcirculation of patients with IBD, and demonstrate the overall importance of the coagulation cascade in intestinal inflammation. Restoring the PC pathway may represent a new therapeutic approach to suppressing intestinal inflammation in IBD, and is also under investigation in several forms of tissue inflammation including asthma, rheumatoid arthritis and atherosclerosis. ${ }^{90}$

A further example of overlap between inflammation and coagulation is represented by the procoagulant molecule tissue factor. Its expression is increased in the microvasculature of the inflamed mucosa, closely correlating with the degree of thrombosis in patients with $\mathrm{CD} .^{84}$ In mice with DSS-induced colitis, antibody blockade of tissue factor prevented several aspects of both coagulation and inflammation, including prevention of the increase in thrombin-antithrombin complexes, reduced leucocyte and platelet recruitment, and tissue injury, as well as blunted thrombus formation. ${ }^{95}$ 
Box 2

Coagulation and inflammation share multiple pathways and are both activated in patients with IBD

- Classical coagulative cells, such as platelets, and coagulative pathways, such as the protein $\mathrm{C}$ pathway and tissue factor, promote intestinal inflammation

- Modulation of coagulative cells and coagulative molecules could offer an entirely new way to treat IBD

Taken together, the above evidence suggests that targeting classically recognised coagulative molecules has a strong impact on inflammation and that coagulation should therefore be actively pursued as a potential target for translational therapy in IBD.

\section{Innate immunity}

The innate immune response is triggered by the binding of pathogen-associated molecular patterns to ubiquitously expressed cell-surface patternrecognition receptors (PRRs). The best studied of the PRRs are the toll-like receptors (TLRs), which can trigger the expression of proinflammatory genes, leucocyte chemotaxis, phagocytosis and cytotoxicity, as well as activating the adaptive immune responses. ${ }^{96}$ It has clearly been established that PRRs are expressed on the surface of cells of the immune system. However, more recently, evidence has started to accumulate that indicates that expression of PRRs on the surface of nonimmune cells, including VECs, plays an important role in the innate immune response. For example, the accumulation of neutrophils may depend on TLR4 expressed on the surface of VECs, rather than leucocytes, as sequestration of neutrophils in the lung is deeply impaired in mice that lack expression of TLR4 in the endothelium. ${ }^{97}$ However, the latter observation contrasts with the significant decrease in leucocyte binding caused by lipopolysaccharide (LPS) in HIMECs. This may reflect tolerance of HIMECs to high concentrations of endotoxin, which they are constantly exposed to in the gut microenvironment. ${ }^{98}$

Expression of TLRs on the surface of VECs is upregulated by vascular inflammation, as well as more specifically by LPS. ${ }^{99}$ However, the expression patterns of different TLRs vary depending on the origin of the VECs. Expression of the bacterial flagellin receptor, TLR5, and the double-stranded

\section{Box 3}

The intestinal endothelium is involved in innate immunity by expressing TLR and nod like receptors (NLR)

- Because of high exposure to bacterial content, the intestinal endothelium is a key second checkpoint for bacterial entry

- Stimulation of its ability to prevent bacterial entry could prevent dissemination of pathogens and bacteria in patients with IBD, where epithelial permeability is increased
RNA receptor, TLR3, has been specifically demonstrated on the surface of HIMECs. ${ }^{100} 101$ Indeed, TLR3 on the surface of HIMECs can modulate the expression of IL-12 and related molecules by these cells. ${ }^{101}$ Activation of TLR5 expressed on the surface of dermal VECs upregulated expression of ICAM-1, although this was not shown in HIMECs. ${ }^{100}$

Other PRRs involved in innate immunity include the nucleotide-binding oligomerisation domains (NODs) 1 and 2, cytosolic proteins that regulate inflammation in response to microbial peptides. ${ }^{102}$ Both NODs are expressed on the surface of VECs and are upregulated in response to LPS and proinflammatory cytokines.

The response of HIMECs to PRR ligands has been compared to that of monocytes and dendritic cells. Production of IL- 6 and IL- 8 by HIMECs was upregulated to a comparable extent to, or greater extent than, the immune cells. HIMECs were also shown to produce MCP-1, RANTES and VEGF, as well as to upregulate ICAM-1 and to enhance the adhesiveness of leucocytes. All of these responses were mediated through activation of NF- $\mathrm{B}$ activation and phosphorylation of p38 and Erk1/2 MAPKs. Interestingly, while the monocytes and dendritic cells showed tolerance after repeated doses of the PRR ligands, with decreased production of IL-8, no evidence of tolerance by the HIMECs was observed, with IL-8 production remaining high. The production of IL-8 by HIMECs was further augmented after pretreatment with TNF, indicating sensitisation to bacterial products. ${ }^{103}$

In apparent contrast, Ogawa et al found that HIMECs developed tolerance in response to pretreatment with endotoxin. ${ }^{98}$ Indeed, leucocyte adhesion in response to LPS was significantly attenuated after pretreatment with LPS for 24-48 h. However, in accordance with the study by Scaldaferri et al, ${ }^{103}$ although LPS pretreatment inhibited expression of E-selectin, VCAM-1, IL-6 and CD86, the expression of ICAM-1, IL-8 and HLA-DR, on the other hand, were not altered. ${ }^{98}$

Taken together, the above data suggest that the intestinal endothelium is also actively involved in innate immunity, acting as a second checkpoint for bacterial entry. This task could be particularly important in the prevention of dissemination of pathogens and bacteria in patients with IBD, where epithelial permeability is increased. ${ }^{104}$

\section{LYMPHATIC ENDOTHELIUM}

The two main tasks performed by the lymphatic vasculature are (1) the drainage of activated inflammatory cells into the draining lymph nodes (DLNs) and (2) complementing the vascular network by transporting extravasated fluid unidirectionally from tissues back to the blood circulation, ${ }^{15} 105$ including drainage of inflammatory elements from inflamed tissues. Mediation of leucocyte exit and clearance of chemokines by the lymphatic vasculature also prevents the development of oedema under 
physiological conditions. ${ }^{15} 106$ These functions underlie the fundamental role that the lymphatic vasculature plays in immunity, ${ }^{17} 105107 \quad 108$ as acquired immune responses are triggered by migration of lymphocytes and antigen-presenting cells through peripheral lymphatic capillaries and into the DLNs. ${ }^{17} 107108$

Given the essential role played by the lymphatics in the resolution of inflammation, it is possible to envisage a critical role for dysfunction of the lymphatic vasculature in the development and/or maintenance of diseases of chronic inflammation such as IBD. However, investigation of a role for the lymphatics at a molecular and cellular level has only recently gained attention, and such a role has yet to be clearly elucidated. It is therefore noteworthy that, since as early as the 1930s, pathologists have reported that the fundamental alteration in the mucosa of patients with CD is consistent with chronic lymphangitis. ${ }^{16}$ Then in the 1970 s it was demonstrated that obstruction of the lymphatics of the small intestine in rats and pigs could generate fistulising intestinal disease, which shared many characteristics with $\mathrm{CD}^{16} 109{ }^{110}$; indeed these models are felt to more closely resemble $\mathrm{CD}$ in humans than any generated subsequently.

Recently the lymphatic system has been identified as a critical controller of experimental colitis and inflammation-associated colon cancer through the decoy receptor D6. Expression of D6 is upregulated on the lymphatic endothelium and intestinal leucocytes of patients with IBD, with a further marked increase in patients with IBDassociated colon cancer. ${ }^{111}$ D6 is involved in the post-inflammatory clearance of $\beta$-chemokines, and deficiency of D6 results in greater inflammation and accumulation of CC chemokines in DSS colitic mice in which D6 had been knocked-out than in wild-type mice; this was also associated with increased leucocytic infiltration. The protective effect of D6 was found to be specifically a function

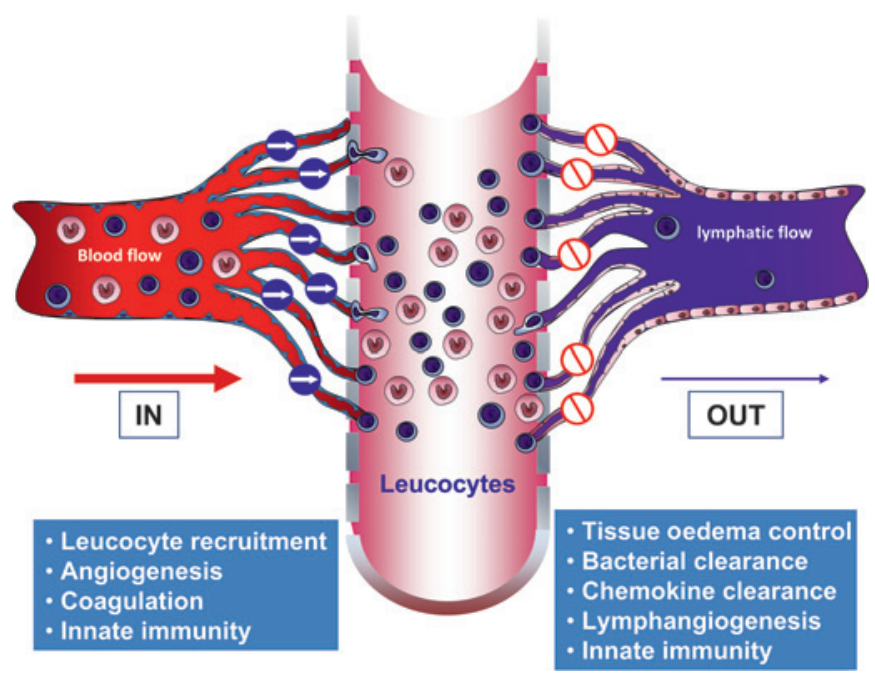

Figure 2 The 'In' and 'Out' of intestinal inflammation. The major tasks performed by the vascular and lymphatic endothelium are summarised. of D6 expression, as disease severity was unchanged in mice deficient in D6 on the lymphatics but with wild-type leucocytes, while mice in which the lymphatics were wild-type for D6, but lacking expression on haematopoietic cells developed disease of the same severity as wild-type mice. These results therefore support a role for the lymphatic system in the control of intestinal inflammation through the decoy receptor D6.

Although researchers have only recently begun investigating the pathogenic role of the lymphatic vasculature in IBD, in other forms of tissue chronic inflammation, such as psoriasis and rheumatoid arthritis, the lymphatic system has received much more attention. For instance, in a recent study, Kataru et al demonstrated expansion of lymphatic vessels in a bacterial pathogen-induced model of acute skin inflammation, which was associated with significantly increased migration of macrophages and other inflammatory cells into the DLNs, as well as an overall increase in the lymph flow. ${ }^{106}$ There was concomitant upregulation of the lymphangiogenic factors, VEGF-A, $-\mathrm{C}$ and $-\mathrm{D}$, blockade of which attenuated the lymphangiogenesis, migration of inflammatory cells, and antigen clearance. On the other hand, migration of inflammatory cells to the DLNs and resolution of inflammation were enhanced in VEGF-C transgenic mice. ${ }^{106}$ These findings clearly indicate the importance of the lymphatic vasculature in the resolution of skin inflammation, and it is likely that similar events could also occur in the gut.

In inflammation, the lymphatic system undergoes intense expansion through lymphangiogenesis. ${ }^{105}$ Lymphangiogenesis occurs in chronically inflamed tissues, such as in the inflamed synovium, psoriatic skin, kidney transplant undergoing rejection, the intima of atherosclerotic lesions, and the lung or corneal inflammation. ${ }^{15} 105112113$ This is true also in patients with IBD, although there are only limited data available regarding the involvement and regulation of lymphangiogenesis, with a few descriptions of aberrant increases in the lymphatic vasculature in patients with $\mathrm{CD}$ and UC. ${ }^{114} 115$ A significant increase in lymphatic vessels has been observed in patients with active UC compared with treated UC, in particular involving extension of lymphatic vessels into areas where they are not normally found under physiological conditions, such as the lamina propria. ${ }^{114}$ The increase in staining for lymphatic vessels was also found to be similar in patients with CD compared with UC. ${ }^{116}$

A comprehensive analysis of the lymphatic system and its functional role in the pathogenesis of IBD is therefore needed, as well as the therapeutic relevance of its blockade or stimulation. Indeed, a recent paper has reported functional changes in the lymphatics in the TNBS-induced model of ileitis in guinea pigs. The investigators found that the lymphatic function of isolated vessels was impaired in colitis compared with control guinea pigs. ${ }^{117}$ A correlation between the degree of inflammation and the functional 
impairment was demonstrated. This manifested in vivo, with a decrease in spontaneous constrictions in the lymphatics of animals in which ileitis had been induced. ${ }^{117}$ However, no data regarding lymphatic function in humans are available thus far.

The isolation and in vitro culture of human intestinal LECs has allowed characterisation of LECs and comparisons with VECs. ${ }^{118}$ Among other important findings, it has now been established that certain markers are unique to LECs compared with VECs. ${ }^{119} 120$ Examples of markers that are unique to LECs compared with VECs include the glomerular podocyte membrane mucoprotein podoplanin, Prox-1, a homeobox gene product that is involved in early lymphatic development, and LYVE-1, a receptor for hyaluronan. ${ }^{119}{ }^{120}$ Definitive data on the characterisation and function of human intestinal LECs will help further our understanding of the role played by the lymphatic system in patients with IBD, keeping in mind the important role played by this system in immunity, bacterial clearance and absorption of oedema. Therefore, stimulating lymphatic function could be therapeutically relevant by promoting the exit of pathogenic leucocytes from the inflamed gut, clearing inflammatory chemokines, and resolving interstitial oedema.

\section{CONCLUSION}

The majority of the research that has been carried out so far into the mechanisms that underlie the development and maintenance of chronic inflammation in patients with IBD has focused on the mechanisms of cell entry into the mucosa, bacterial and foreign antigen invasion, angiogenesis, and the control of gut inflammation through intestinal microvasculature. These mechanisms, the 'IN' of chronic inflammation in patients with IBD (figure 2), have provided several valid and effective targets, and promise many more-both on immune and non-immune cells-for the treatment of experimental inflammation, some of which have translated into patients. However, it is becoming increasingly clear that it is also important to focus on the 'OUT' of chronic inflammation, by studying the lymphatics and their role in controlling tissue oedema, leucocyte exit, bacterial clearance and oedema absorption, as this promises to be another rich source of non-immune cell targets for therapeutic intervention. The functions of the microvascular and lymphatic endothelium in the gut are therefore complementary, although opposite. In patients with IBD, a dysfunction in each would work together to amplify disease, with the microvascular endothelium overloading, and the lymphatic endothelium failing to relieve the intestinal mucosa. Just as one needs the other for proper physiological function, so dysfunction in one may support dysfunction in the other in disease pathogenesis, the proverbial 'brother in arms'.

Acknowledgements I thank Sarah A De La Rue of Readable Science for her assistance with the manuscript.
Funding The studies reported in this review were supported by grants from the Broad Medical Research Program, the Italian Ministry of Health (Ricerca Finalizzata 2006, n.72 and Bando Giovani Ricercatori), Fondazione Cariplo, the Italian Association for Cancer Research (my first AIRC Grant and IG 10205) and AMICI Italia to SD. This work was conducted in the context, and with the support, of the Fondazione Humanitas per la Ricerca (Rozzano, Italy).

Competing interests None.

Provenance and peer review Commissioned; externally peer reviewed.

\section{REFERENCES}

1. Fiocchi C. Intestinal inflammation: a complex interplay of immune and nonimmune cell interactions. Am J Physiol 1997:273:G769-75.

2. Danese S, Fiocchi C. Etiopathogenesis of inflammatory bowel diseases. World J Gastroenterol 2006;12:4807-12.

3. Fiocchi C. From immune activation to gut tissue injury: the pieces of the puzzle are coming together. Gastroenterology 1999:117:1238-41.

4. Danese S. Inflammation and the mucosal microcirculation in inflammatory bowel disease: the ebb and flow. Curr Opin Gastroenterol 2007:23:384-9.

5. Danese S, Papa A, Saibeni S, et al. Inflammation and coagulation in inflammatory bowel disease: The clot thickens. Am J Gastroenterol 2007:102:174-6.

6. Carmeliet P. Angiogenesis in health and disease. Nat Med 2003;9:653-60.

7. Rieder $\mathbf{F}$, Brenmoehl J, Leeb S, et al. Wound healing and fibrosis in intestinal disease. Gut 2007; 56:130-9.

8. Andoh A, Bamba S, Brittan M, et al. Role of intestina subepithelial myofibroblasts in inflammation and regenerative response in the gut. Pharmacol Ther 2007;114:94-106.

9. Danese $\mathbf{S}$. Non-immune cells in inflammatory bowel disease: from victim to villain. Trends Immunol 2008;29:555-64.

10. Danese $\mathbf{S}$, Dejana $\mathrm{E}$, Fiocchi $\mathrm{C}$. Immune regulation by microvascular endothelial cells: directing innate and adaptive immunity, coagulation, and inflammation. J Immunol 2007:178:6017-22.

11. Granger DN, Kubes $P$. The microcirculation and inflammation: modulation of leukocyte-endothelial cell adhesion. J Leukoc Biol 1994:55:662-75.

12. Hatoum $\mathbf{0 A}$, Miura $\mathrm{H}$, Binion DG. The vascular contribution in the pathogenesis of inflammatory bowel disease. Am J Physiol Heart Circ Physiol 2003;285:H1791-6.

13. Cines DB, Pollak ES, Buck CA, et al. Endothelial cells in physiology and in the pathophysiology of vascular disorders. Blood 1998:91:3527-761.

14. Deban L, Correale C, Vetrano S, et al. Multiple pathogenic roles of microvasculature in inflammatory bowel disease: a Jack of all trades. Am J Pathol 2008;172:1457-66.

15. Saharinen P, Tammela T, Karkkainen MJ, et al. Lymphatic vasculature: development, molecular regulation and role in tumo metastasis and inflammation. Trends Immunol 2004;25:387-95.

16. Van Kruiningen HJ, Colombel JF. The forgotten role of lymphangitis in Crohn's disease. Gut 2008:57:1-4.

17. Lawrence T, Gilroy DW. Chronic inflammation: a failure of resolution? Int J Exp Pathol 2007:88:85-94.

18. Ades EW, Candal FJ, Swerlick RA, et al. HMEC-1: establishment of an immortalized human microvascular endothelial cell line. J Invest Dermatol 1992:99:683-90.

19. Binion DG, West GA, Ina K, et al. Enhanced leukocyte binding by intestinal microvascular endothelial cells in inflammatory bowel disease. Gastroenterology 1997:112:1895-907.

20. Kvietys PR, Granger DN. Endothelial cell monolayers as a tool for studying microvascular pathophysiology. Am J Physiol 1997:273 G1189-99.

21. Aird WC, Edelberg JM, Weiler-Guettler $\mathrm{H}$, et al. Vascular bedspecific expression of an endothelial cell gene is programmed by the tissue microenvironment. J Cell Biol 1997;138:1117-24.

22. Granger DN. Cell adhesion and migration. II. Leukocyteendothelial cell adhesion in the digestive system. Am J Physiol 1997;273:G982-6

23. Panes J, Granger DN. Leukocyte-endothelial cell interactions: molecular mechanisms and implications in gastrointestinal disease. Gastroenterology 1998;114:1066-90.

24. Danese S, Semeraro S, Marini M, et al. Adhesion molecules in inflammatory bowel disease: therapeutic implications for gut inflammation. Dig Liver Dis 2005;37:811-18. 
25. Fiorino G, Correale C, Fries W, et al. Leukocyte traffic control: a novel therapeutic strategy for inflammatory bowel disease. Expert Rev Clin Immunol 2010;6:567-72.

26. Rivera-Nieves J, Gorfu G, Ley K. Leukocyte adhesion molecules in animal models of inflammatory bowel disease. Inflamm Bowel Dis 2008:14:1715-35

27. Stefanelli T, Malesci A, De La Rue SA, et al. Anti-adhesion molecule therapies in inflammatory bowel disease: touch and go. Autoimmun Rev 2008:7:364-9.

28. Papadakis KA, Prehn J, Moreno ST, et al. CCR9-positive lymphocytes and thymus-expressed chemokine distinguish small bowel from colonic Crohn's disease. Gastroenterology 2001;121:246-54.

29. Apostolaki M, Manoloukos M, Roulis M, et al. Role of beta7 integrin and the chemokine/chemokine receptor pair CCL25/CCR9 in modeled TNF-dependent Crohn's disease. Gastroenterology 2008:134:2025-35.

30. Rivera-Nieves J, Ho J, Bamias G, et al. Antibody blockade of CCL25/CCR9 ameliorates early but not late chronic murine ileitis Gastroenterology 2006:131:1518-29.

31. Binion DG, West GA, Volk EE, et al. Acquired increase in leucocyte binding by intestinal microvascular endothelium in inflammatory bowel disease. Lancet 1998;352:1742-6.

32. Briskin M, Winsor-Hines D, Shyjan A, et al. Human mucosal addressin cell adhesion molecule-1 is preferentially expressed in intestinal tract and associated lymphoid tissue. Am J Pathol 1997;151:97-110.

33. Rivera-Nieves J, Olson T, Bamias G, et al. L-selectin, alpha 4 beta 1 , and alpha 4 beta 7 integrins participate in $\mathrm{CD} 4+\mathrm{T}$ cell recruitment to chronically inflamed small intestine. J Immunol 2005;174:2343-52

34. Danese S, Sans M, Scaldaferri F, et al. TNF-alpha blockade downregulates the CD40/CD40L pathway in the mucosal microcirculation: a novel anti-inflammatory mechanism of infliximab in Crohn's disease. J Immunol 2006;176:2617-24.

35. Sans M, Danese S, De La Motte C, et al. Enhanced recruitment of CX3CR1 + T cells by mucosal endothelial cell-derived fractalkine in inflammatory bowel disease. Gastroenterology 2007:132:139-53

36. Danese S, Sans M, Fiocchi C. The CD40/CD40L costimulatory pathway in inflammatory bowel disease. Gut 2004;53:1035-43.

37. Danese S, De La Motte C, Sturm A, et al. Platelets trigger a CD40-dependent inflammatory response in the microvasculature of inflammatory bowel disease patients. Gastroenterology 2003:124:1249-64.

38. Danese S, Katz J, Saibeni S, et al. Activated platelets are the source of elevated levels of soluble CD40 ligand in the circulation of inflammatory bowel disease patients. Gut 2003;10:1435-41.

39. Danese S, De La Motte C, Reyes BM, et al. Cutting edge: T cells trigger CD40-dependent platelet activation and granular RANTES release: a novel pathway for immune response amplification. $J$ Immunol 2004:172:2011-15.

40. Vogel JD, West GA, Danese S, et al. CD40-mediated immune-nonimmune cell interactions induce mucosal fibroblast chemokines leading to T-cell transmigration. Gastroenterology 2004; 126:63-80

41. Vowinkel T, Anthoni C, Wood KC, et al. CD40-CD40 Ligand mediates the recruitment of leukocytes and platelets in the inflamed murine colon. Gastroenterology 2007;132:955-65.

42. Krieglstein CF, Cerwinka WH, Laroux FS, et al. Regulation of murine intestinal inflammation by reactive metabolites of oxygen and nitrogen: divergent roles of superoxide and nitric oxide. J Exp Med 2001:194:1207-18.

43. Binion DG, Rafiee P, Ramanujam KS, et al. Deficient iNOS in inflammatory bowel disease intestinal microvascular endothelial cells results in increased leukocyte adhesion. Free Radic Biol Med 2000;29:881-8.

44. Binion DG, Fu S, Ramanujam KS, et al. iNOS expression in human intestinal microvascular endothelial cells inhibits leukocyte adhesion. Am J Physiol 1998;275:G592-603.

45. Horowitz S, Binion DG, Nelson V, et al. Increased arginase activity and endothelial dysfunction in human inflammatory bowel disease. Am J Physiol 2007;292:G1323-36.

46. Danese S, Sgambato A, Papa A, et al. Homocysteine triggers mucosal microvascular activation in inflammatory bowel disease. Am J Gastroenterol 2005;100:886-95.

47. Scaldaferri F, Sans M, Vetrano $S$, et al. The role of MAPK in governing lymphocyte adhesion to and migration across the microvasculature in inflammatory bowel disease. Eur $\mathrm{J}$ Immunol 2009:39:290-300.
48. Scaldaferri F, Correale C, Gasbarrini A, et al. Molecular signaling blockade as a new approach to inhibit leukocyte-endothelial interactions for inflammatory bowel disease treatment. Cell Adh Migr 2009:3:296-9.

49. Hommes D, van den Blink B, Plasse T, et al. Inhibition of stressactivated MAP kinases induces clinical improvement in moderate to severe Crohn's disease. Gastroenterology 2002;122:7-14.

50. Jackson JR, Seed MP, Kircher CH, et al. The codependence of angiogenesis and chronic inflammation. FASEB J

1997:11:457-65.

51. Koutroubakis IE, Tsiolakidou G, Karmiris K, et al. Role of angiogenesis in inflammatory bowel disease. Inflamm Bowel Dis 2006;12:515-23.

52. Chidlow JH Jr, Shukla D, Grisham MB, et al. Pathogenic angiogenesis in IBD and experimental colitis: new ideas and therapeutic avenues. Am J Physiol Gastrointest Liver Physiol 2007:293:G5-18.

53. Rutella S, Vetrano S, Correale C, et al. Enhanced platelet adhesion induces angiogenesis in intestinal inflammation and inflammatory bowel disease microvasculature. J Cell Mol Med 2011;15:625-34.

54. Milkiewicz M, Brown MD, Egginton S, et al. Association between shear stress, angiogenesis, and VEGF in skeletal muscles in vivo. Microcirculation 2001;8:229-41.

55. Cane G, Moal VL, Pages G, et al. Up-regulation of intestinal vascular endothelial growth factor by Afa/Dr diffusely adhering Escherichia coli. PLoS One 2007;2:e1359.

56. Im E, Choi YJ, Kim CH, et al. The angiogenic effect of probiotic Bacillus polyfermenticus on human intestinal microvascular endothelial cells is mediated by IL-8. Am J Physiol Gastrointest Liver Physiol 2009;297:G999-1008.

57. Carmeliet $\mathbf{P}$. Mechanisms of angiogenesis and arteriogenesis. Nat Med 2000;6:389-95.

58. Majno G. Chronic inflammation: links with angiogenesis and wound healing. Am J Pathol 1998;153:1035-9.

59. Danese S, Sans M, De La Motte C, et al. Angiogenesis as a novel component of inflammatory bowel disease pathogenesis. Gastroenterology 2006;130:2060-73

60. Bardin N, Reumaux D, Geboes K, et al. Increased expression of CD146, a new marker of the endothelial junction in active inflammatory bowel disease. Inflamm Bowel Dis 2006;12:16-21.

61. Saito S, Tsuno NH, Sunami E, et al. Expression of plateletderived endothelial cell growth factor in inflammatory bowel disease. J Gastroenterol 2003;38:229-37.

62. Kanazawa S, Tsunoda T, Onuma E, et al. VEGF, basic-FGF, and TGF-beta in Crohn's disease and ulcerative colitis: a novel mechanism of chronic intestinal inflammation. Am J Gastroenterol 2001:96:227-8.

63. Bousvaros A, Zurakowski D, Fishman SJ, et al. Serum basic fibroblast growth factor in pediatric Crohn's disease. Implications for wound healing. Dig Dis Sci 1997:42:378-86.

64. Koutroubakis IE, Xidakis $\mathrm{C}$. Karmiris $\mathrm{K}$, et al. Potential role of soluble angiopoietin-2 and Tie-2 in patients with inflammatory bowel disease. Eur J Clin Invest 2006;36:127-32.

65. Ferrante M, Pierik M, Henckaerts L, et al. The role of vascular endothelial growth factor (VEGF) in inflammatory bowel disease. Inflamm Bowel Dis 2006:12:870-8.

66. Ganta VC, Cromer W, Mills GL, et al. Angiopoietin-2 in experimental colitis. Inflamm Bowel Dis 2010;16:1029-39.

67. Oikonomou KA, Kapsoritakis AN, Kapsoritaki Al, et al. Angiogenin, angiopoietin-1, angiopoietin-2, and endostatin serum levels in inflammatory bowel disease. Inflamm Bowel Dis 2011:17:963-70.

68. Im E, Rhee SH, Park YS, et al. Corticotropin-releasing hormone family of peptides regulates intestinal angiogenesis. Gastroenterology 2010:138:2457-67. 2467.e1-5.

69. Koon HW, Zhao D, Xu H, et al. Substance P-mediated expression of the pro-angiogenic factor CCN1 modulates the course of colitis. Am J Pathol 2008;173:400-10.

70. Danese S, Scaldaferri F, Vetrano S, et al. Critical role of the CD40-CD40 ligand pathway in governing mucosal inflammationdriven angiogenesis in inflammatory bowel disease. Gut 2007:56:1248-56.

71. Goebel S, Huang M, Davis WC, et al. VEGF-A stimulation of leukocyte adhesion to colonic microvascular endothelium: implications for inflammatory bowel disease. Am J Physiol 2006:290:G648-54.

72. Chidlow JH Jr, Langston W, Greer JJ, et al. Differential angiogenic regulation of experimental colitis. Am J Pathol 2006:169:2014-30. 
73. Danese S, Sans M, Spencer DM, et al. Angiogenesis blockade as a new therapeutic approach to experimental colitis. Gut 2007:56:855-62.

74. Scaldaferri F, Vetrano S, Sans M, et al. VEGF-A links angiogenesis and inflammation in inflammatory bowel disease pathogenesis. Gastroenterology 2009;136:585-95.

75. Tolstanova G, Khomenko T, Deng $X$, et al. Neutralizing antivascular endothelial growth factor (VEGF) antibody reduces severity of experimental ulcerative colitis in rats: direct evidence for the pathogenic role of VEGF. J Pharmacol Exp Ther 2009:328:749-57.

76. Chidlow JH Jr, Greer JJ, Anthoni C, et al. Endothelial caveolin-1 regulates pathologic angiogenesis in a mouse model of colitis. Gastroenterology 2009;136:575-84.

77. Punekar S, Zak S, Kalter VG, et al. Thrombospondin 1 and its mimetic peptide ABT-510 decrease angiogenesis and inflammation in a murine model of inflammatory bowel disease. Pathobiology 2008;75:9-21.

78. Danese S. Negative regulators of angiogenesis in inflammatory bowel disease: thrombospondin in the spotlight. Pathobiology 2008;75:22-4

79. Adenis A, Vanseymortier L, Foissey D, et al. Bevacizumab and postponed suture leakages after surgery for ulcerative colitis and rectal cancer. Gut 2007:56:734.

80. Loriot Y, Boudou-Rouquette P, Billemont B, et al. Acute exacerbation of hemorrhagic rectocolitis during antiangiogenic therapy with sunitinib and sorafenib. Ann Oncol 2008;19:1975

81. Hindryckx $\mathbf{P}$, Waeytens A, Laukens D, et al. Absence of placental growth factor blocks dextran sodium sulfate-induced colonic mucosal angiogenesis, increases mucosal hypoxia and aggravates acute colonic injury. Lab Invest 2010:90:566-76.

82. Esmon CT. Interactions between the innate immune and blood coagulation systems. Trends Immunol 2004;25:536-42.

83. Danese S, De La Motte C, Fiocchi C. Platelets in Inflammatory bowel disease: clinical, pathological and therapeutical implications. Am J Gastroenterol 2004:99:938-45.

84. Sankey EA, Dhillon AP, Anthony A, et al. Early mucosal changes in Crohn's disease. Gut 1993;34:375-81.

85. Thompson NP, Wakefield AJ, Pounder RE. Inherited disorders of coagulation appear to protect against inflammatory bowel disease. Gastroenterology 1995;108:1011-15.

86. Dhillon AP, Anthony A, Sim R, et al. Mucosal capillary thrombi in rectal biopsies. Histopathology 1992;21:127-33.

87. Mori M, Salter JW, Vowinkel T, et al. Molecular determinants of the prothrombogenic phenotype assumed by inflamed colonic venules. Am J Physiol 2005;288:G920-6.

88. Vowinkel T, Wood KC, Stokes KY, et al. Mechanisms of platelet and leukocyte recruitment in experimental colitis. Am J Physiol 2007;293:G1054-60.

89. Strukova S. Blood coagulation-dependent inflammation Coagulation-dependent inflammation and inflammationdependent thrombosis. Front Biosci 2006;11:59-80.

90. Danese $\mathbf{S}$, Vetrano $S$, Zhang $L$, et al. The protein $C$ pathway in tissue inflammation and injury: pathogenic role and therapeutic implications. Blood 2010;115:1121-30.

91. Lust M, Vulcano M, Danese $\mathrm{S}$. The protein $\mathrm{C}$ pathway in inflammatory bowel disease: the missing link between inflammation and coagulation. Trends $\mathrm{Mol}$ Med 2008;14:237-44.

92. Scaldaferri $\mathbf{F}$, Sans $M$, Vetrano $S$, et al. Crucial role of the protein $C$ pathway in governing microvascular inflammation in inflammatory bowel disease. J Clin Invest 2007;117:1951-60.

93. Faioni EM, Ferrero S, Fontana G, et al. Expression of endothelial protein $\mathrm{C}$ receptor and thrombomodulin in the intestinal tissue of patients with inflammatory bowel disease. Crit Care Med 2004:32:S266-70.

94. Yoshida H, Russell J, Stokes KY, et al. Role of the protein C pathway in the extraintestinal thrombosis associated with murine colitis. Gastroenterology 2008:135:882-8.

95. Anthoni C, Russell J, Wood KC, et al. Tissue factor: a mediator of inflammatory cell recruitment, tissue injury, and thrombus formation in experimental colitis. J Exp Med 2007:204:1595-601.

96. Iwasaki A, Medzhitov R. Toll-like receptor control of the adaptive immune responses. Nat Immunol 2004:5:987-95.
97. Andonegui G, Bonder CS, Green F, et al. Endothelium-derived Toll-like receptor- 4 is the key molecule in LPS-induced neutrophil sequestration into lungs. J Clin Invest 2003;111:1011-20.

98. Ogawa H, Rafiee P, Heidemann J, et al. Mechanisms of endotoxin tolerance in human intestinal microvascular endothelia cells. J Immunol 2003:170:5956-64.

99. Faure $\mathbf{E}$, Thomas $\mathrm{L}, \mathrm{Xu} \mathrm{H}$, et al. Bacterial lipopolysaccharide and IFN-gamma induce Toll-like receptor 2 and Toll-like receptor 4 expression in human endothelial cells: role of NF-kappa B activation. J Immunol 2001;166:2018-24.

100. Maaser C, Heidemann J, von Eiff C, et al. Human intestinal microvascular endothelial cells express Toll-like receptor 5 : a binding partner for bacterial flagellin. $J$ Immunol 2004;172:5056-62.

101. Heidemann J, Ruther C, Kebschull M, et al. Expression of IL-12 related molecules in human intestinal microvascular endothelial cells is regulated by TLR3. Am J Physiol Gastrointest Liver Physio 2007;293:G1315-24.

102. Inohara N, Nunez G. NODs: intracellular proteins involved in inflammation and apoptosis. Nat Rev Immunol 2003:3:371-82.

103. Scaldaferri F, Phillips $M$, Rhee $M$, et al. Tolerization of intestinal mesenchymal and endothelial cells to bacterial products and TNF-á: a novel mechanism to sustain and amplify gut inflammation. Gastroenterology 2009;136:T1691.

104. Heidemann J, Domschke W, Kucharzik T, et al. Intestinal microvascular endothelium and innate immunity in inflammatory bowel disease: a second line of defense? Infect Immun 2006:74:5425-32

105. Alitalo K, Tammela T, Petrova TV. Lymphangiogenesis in development and human disease. Nature 2005;438:946-53.

106. Kataru RP, Jung $\mathrm{K}$, Jang $\mathrm{C}$, et al. Critical role of CD11b+ macrophages and VEGF in inflammatory lymphangiogenesis, antigen clearance, and inflammation resolution. Blood 2009:113:5650-9.

107. Angeli V, Randolph GJ. Inflammation, lymphatic function, and dendritic cell migration. Lymphat Res Biol 2006:4:217-28.

108. Aloisi F, Pujol-Borrell R. Lymphoid neogenesis in chronic inflammatory diseases. Nat Rev Immunol 2006:6:205-17.

109. Kalima TV. Experimental lymphatic obstruction in the ileum. Ann Chir Gynaecol Fenn 1970;59:187-201.

110. Kalima TV, Saloniemi H, Rahko T. Experimental regional enteritis in pigs. Scand J Gastroenterol 1976;11:353-62.

111. Vetrano S, Borroni EM, Sarukhan A, et al. The lymphatic system controls intestinal inflammation and inflammation-associated colon cancer through the chemokine decoy receptor D6. Gut 2009:59:197-206.

112. Mouta C, Heroult M. Inflammatory triggers of lymphangiogenesis. Lymphat Res Biol 2003;1:201-18.

113. Alitalo K, Carmeliet P. Molecular mechanisms of lymphangiogenesis in health and disease. Cancer Cell 2002;1:219-27.

114. Fogt F, Pascha TL, Zhang PJ, et al. Proliferation of D2-40expressing intestinal lymphatic vessels in the lamina propria in inflammatory bowel disease. Int $\mathrm{J} \mathrm{Mol} \mathrm{Med}$ 2004:13:211-14.

115. Wu TF, MacNaughton WK, von der Weid PY. Lymphatic vesse contractile activity and intestinal inflammation. Mem Inst Oswaldo Cruz 2005;100(Suppl 1):107-10.

116. Geleff S, Schoppmann SF, Oberhuber G. Increase in podoplaninexpressing intestinal lymphatic vessels in inflammatory bowel disease. Virchows Arch 2003;442:231-7.

117. Wu TF, Carati CJ, MacNaughton WK, et al. Contractile activity of lymphatic vessels is altered in the TNBS model of guinea pig ileitis. Am J Physiol Gastrointest Liver Physiol 2006;291: G566-74

118. Correale C, Vetrano S, Tognoli F, et al. Lymphoneogenesis: a new component in inflammatory bowel disease pathogenesis. Gastroenterology 2008:134:A656.

119. Podgrabinska S, Braun P, Velasco P, et al. Molecular characterization of lymphatic endothelial cells. Proc Natl Acad Sci U S A 2002:99:16069-74.

120. Amatschek S, Kriehuber E, Bauer W, et al. Blood and lymphatic endothelial cell-specific differentiation programs are stringently controlled by the tissue environment. Blood 2007:109:4777-85. 